activation during the acute phase of KD. Although the drug of choice for treatment of $\mathrm{KD}$ is still intravenous immunoglobulin (IVIg), approximately $10-15 \%$ patients may not respond to the first dose of IVIg. In such cases, an additional dose of IVIg can be given. Infliximab, a TNF- $\alpha$ inhibitor, is also being increasingly used in such situations. We present our experience with use of infliximab in 9 patients with refractory $\mathrm{KD}$ at a tertiary care cente.

Patients and methods We diagnosed 112 children with KD during the period January 2007 - November 2012. The diagnosis of $\mathrm{KD}$ was based on the American Heart Association criteria. All children underwent detailed echocardiography examinations by a paediatric cardiologist. Standard treatment protocols (IVIg $2 \mathrm{~g} / \mathrm{kg}$ along with aspirin) were used in the management of these children. In addition, 9 children with $\mathrm{KD}$ received infliximab (REMICADE) during this period. The drug was used in doses of $5-7 \mathrm{mg} / \mathrm{kg}$ and given intravenously,. Indicationas for using infliximab were as follows: refractory KD in 6 children; 3 children had come in late ( $>3$ weeks) and had coronary artery abnormalities (CAA) at presentation. 7 patients had developed CAA abnormalities before infliximab therapy: transient mild dilatation-2; aneurysms-3; giant aneurysms-2.

Results Complete response to therapy with cessation of fever occurred in 7/9 patients. Platelet count and C-reactive protein concentrations decreased following infliximab infusion in all, except 2. The latter 2 patients, on follow-up, progressed and developed features consistent with systemic onset juvenile idiopathic arthritis. 4 children with mild CAA (dilatation/small aneurysms) showed complete resolution on follow-up; 2 children with giant aneurysms showed no further progression. No side-effects were noted and there were no significant infusion reactions.

Conclusion Infliximab may have a role in refractory $\mathrm{KD}$, especially when there are CAA. In older children, the use of infliximab is also more economical than IVIg. This has therapeutic implications in resource constrained settings of a developoing country.

\section{G233 AORTIC WALL STIFFNESS STUDIES IN CHILDREN WITH KAWASAKI DISEASE WITHOUT CORONARY ARTERY ABNORMALITIES - A FOLLOW-UP STUDY FROM NORTH INDIA}

doi:10.1136/archdischild-2013-304107.245

'A Gupta, 'S Singh, 'A Gupta, 'D Suri, ${ }^{2 R}$ Manojkumar. 'Paediatrics, Post Graduate Institute of Medical Education and Research, Chandigarh, India; ${ }^{2}$ Cardiology, Post Graduate Institute of Medical Education and Research, Chandigarh, India

Introduction The main focus of concern in Kawasaki disease (KD) hitherto has been on children who develop coronary artery abnormalities (CAA). There is emerging evidence to suggest that several long term cardiovascular sequelae (e.g. endothelial dysfunction), can occur in children with KD even without CAA. Abdominal aortic wall stiffness (AWS) is a surrogate marker of endothelial dysfunction. We evaluated AWS in a cohort of 30 North Indian children with $\mathrm{KD}$ without CAA, at least 5 years after the acute phase. There is paucity of literature on follow-up studies of AWS in $\mathrm{KD}$ in this setting.

Patients and methods 30 children with $\mathrm{KD}$ without CAA, diagnosed at least 5 years back, and who had a normal echocardiography examination at time of enrolment were included in the study. The diagnosis of $\mathrm{KD}$ was made on basis of American Heart Association criteria. Age, sex and socio-economic status matched healthy children were included as controls. All children underwent abdominal aorta studies by an experienced cardiologist (RM). Clinical measurements were obtained on Siemens Acuson Sequoia C512 Echocardiography Machine. The study protocol was approved by Institute Ethics Committee.
Results Mean age of cases was $11.24 \pm 3.48$ years with a mean interval of $7.72 \pm 2.37$ years from initial diagnosis of $\mathrm{KD}$. Mean aortic strain was $0.345 \pm 0.145$ in cases as against $0.369 \pm 0.123$ in controls ( $p>0.05$ ). Mean pressure strain elastic modulus (SEM) was $124.454 \pm 57.052 \mathrm{~N} / \mathrm{m}^{2}$ in cases as against $112.526 \pm 48.752$ in controls $(p>0.05)$. Mean normalised pressure SEM was $1.898 \pm 1.023$ in cases as against $1.700 \pm 0.700$ in controls $(p>0.05)$. Mean peak flow velocity was $0.931 \pm 0.277 \mathrm{~m} / \mathrm{sec}$ in cases and $0.915 \pm 0.215$ in controls. No statistically significant differences could be detected between cases and controls in terms of aortic root diameters.

Conclusion Though a distinct trend towards higher AWS and lower distensibility was discernible in children with KD (without CAA), the difference did not achieve statistical significance. This could be because of the small sample size in this study. More longterm studies on a greater number of subjects are needed to ascertain the extent of endothelial dysfunction in children with $\mathrm{KD}$ without CAA. This may have implications on long-term prognostication of children with KD without CAA.

\section{G234 ACUTE TRANSVERSE MYELITIS AS THE INITIAL MANIFESTATION OF SYSTEMIC LUPUS ERYTHEMATOSUS IN A CHILD}

doi:10.1136/archdischild-2013-304107.246

'V Shivamurthy, ${ }^{2} \mathrm{~S}$ Ganesan, ${ }^{2} \mathrm{~A}$ Khan, ${ }^{1} \mathrm{~A}$ Sridhar, ${ }^{2} \mathrm{~N}$ Hussain. ${ }^{1}$ Paediatrics, University Hospitals of Leicester NHS Trust, Leicester, UK; ${ }^{2}$ Paediatric Neurology, University Hospitals of Leicester, Leicester, UK

Introduction Systemic Lupus Erthematosus (SLE) is a chronic autoimmune disease characterised by multisystem involvement. It is complicated by neurological manifestations in $25-95 \%$ of the patients. Acute transverse myelitis may be a complication in 1-2\% of patients but in some it may even be the initial manifestation of SLE.

Case report 13 years old previously healthy girl was admitted with a history of pain in her lower limbs for 2 weeks, fever for 2 days and sensory loss. She developed acute flaccid quadriparesis over the next $48 \mathrm{hrs}$, with bilateral weakness of her lower limbs, urinary retention and sensory loss from T2 spinal segment down for light touch and pain. Her vibratory and proprioception were absent in her lower limbs. Power in her upper limbs was reduced. She had no cranial nerve involvement and her ophthalmology assessment was unremarkable. She did not have bulbar involvement or respiratory difficulties. Investigations revealed raised inflammatory markers and CSF showed pleocytosis with an elevated protein. She didn't fulfil the ACR criteria for SLE but her blood results were consistent for an active SLE (ANA positive speckled pattern, double stranded DNA, Anti Sm and Anti RNP antibody positive with low complement C3 and C4 level). Her cultures for blood, urine, CSF were negative as well as extensive virology screen and serology for Lyme's disease. MRI Spine showed high signal within the spinal cord from C5 down to the conus suggestive of multiregional multifocal transverse myelitis. Her MRI brain was normal. She was treated with 7 cycles of cyclophosphamide and methylprednisolone and plasmapharesis. She was wheelchair bound but has responded well to the treatment and is able to walk few steps without support, manage with crutches only to walk and only uses wheelchair occasionally. She doesn't have any upper limb problems at the moment although still has neuropathic bladder.

Conclusion It is important to include autoimmune screen as part of transverse myelitis work up as SLE may not present with typical clinical features at the outset.

Early diagnosis and aggressive treatment of transverse myelitis is important in SLE to prevent long-term disability. 